\title{
Dominant Local Binary Patterns for Texture Classification
}

\author{
S. Liao, Max W. K. Law, and Albert C. S. Chung
}

\begin{abstract}
This paper proposes a novel approach to extract image features for texture classification. The proposed features are robust to image rotation, less sensitive to histogram equalization and noise. It comprises of two sets of features: dominant local binary patterns (DLBP) in a texture image and the supplementary features extracted by using the circularly symmetric Gabor filter responses. The dominant local binary pattern method makes use of the most frequently occurred patterns to capture descriptive textural information, while the Gabor-based features aim at supplying additional global textural information to the DLBP features. Through experiments, the proposed approach has been intensively evaluated by applying a large number of classification tests to histogram-equalized, randomly rotated and noise corrupted images in Outex, Brodatz, Meastex, and CUReT texture image databases. Our method has also been compared with six published texture features in the experiments. It is experimentally demonstrated that the proposed method achieves the highest classification accuracy in various texture databases and image conditions.
\end{abstract}

Index Terms-Circularly symmetric Gabor filter, local binary pattern, rotation invariance, texture classification.

\section{INTRODUCTION}

$\mathbf{T}$ EXTURE classification plays an important role in computer vision and image processing applications. The applications include medical image analysis and understanding, remote sensing, object-based image coding, and image retrieval. As the demand of such applications increases, texture classification has received considerable attention over the last several decades and numerous novel methods have been proposed.

For example, Chellappa et al. used the Gaussian Markov random fields (GMRF) to model texture patterns based on statistical relationship between adjacent pixel intensity values [4]. Bovik et al. applied the Gabor filters to an image and then computed the average filter responses as features [2]. Mallat introduced the multiresolution wavelet decomposition method, which generates coefficients in the HL, LH, and LL channels for subsequent classification tasks [18]. Weszka et al. [23] applied the co-occurrence matrix to extract the mean intensity, contrast, and correlation information from the texture images.

Manuscript received December 07, 2007; revised December 11, 2008. Current version published April 10, 2009. The associate editor coordinating the review of this manuscript and approving it for publication was Prof. Dan Schonfeld.

The authors are with the Lo Kwee-Seong Medical Image Analysis Laboratory, Department of Computer Science and Engineering, The Hong Kong University of Science and Technology, Clear Water Bay, Hong Kong.

Digital Object Identifier 10.1109/TIP.2009.2015682
However, the above techniques only encode the absolute texture orientation information, which is inadequate for rotation invariant texture classification.

Therefore, to achieve rotation invariance in texture classification, researchers have attempted to either discard all orientation information or capture relative orientation information. To discard orientation information, Porter and Canagarajah [20] removed the $\mathrm{HH}$ wavelet channels and combined the LH and HL wavelet channels to obtain rotation invariant wavelet features. Haley and Manjunath [8] calculated isotropic rotation invariant features from Gabor filters. Kashyap and Khotanzad [12] constructed an isotropic circular Gaussian Markov random field (ICGMRF). On the other hand, some approaches capture the relative directional features rather than the absolute orientation information. Deng and Clausi [6] extended the ICGMRF model [12] into anisotropic circular GMRF model (ACGMRF) to capture rotation invariant relative orientation features. Along the same research line, Arof and Deravi [1] utilized similar circular neighborhoods with 1-D DFT transformation.

Although the aforementioned methods are proofed to be rotation invariant, they are sensitive to the change of illumination condition which often exists in texture images because of the limitation of the imaging devices or the change of lighting condition. In real world applications, histogram equalization is often performed to mitigate the adverse effect of varying illumination condition. However, as we will show in the Experiments and Results Section (Section IV), the performances of the above methods drop significantly after the histogram equalization has been applied because, for these methods, intensity mean and image contrast are two important pieces of textural information for texture classification. Ojala et al. [19] proposed rotation and histogram equalization invariant features by observing the statistical distributions of the uniform local binary patterns (LBPs). Huang et al. extended the LBP method by calculating the derivative-based LBPs in the application of face alignment [9]. However, the uniform LBPs are not the dominating patterns (i.e., patterns of the largest proportions in an image) in some textures with irregular edges and shapes. This observation will be further elaborated in Section II.

In this paper, we are motivated to propose a new feature extraction method that is robust to histogram equalization and rotation. First, the conventional LBP approach is extended to the dominant local binary pattern (DLBP) approach in order to effectively capture the dominating patterns in texture images. Unlike the conventional LBP approach, which only exploits the uniform LBP, given a texture image, the DLBP approach computes the occurrence frequencies of all rotation invariant patterns defined in the LBP groups. These patterns are then sorted in descending order. The first several most frequently occurring 
patterns should contain dominating patterns in the image and, therefore, are the dominant patterns. It is shown that the DLBP approach is more reliable to represent the dominating pattern information in the texture images.

Although the DLBP features encapsulate more textural information than the conventional LBP features, they lack the consideration of distant pixel interactions. The reason is that the binary patterns are extracted in the proximity of local pixels. The pixel interaction that takes place outside the local neighborhood system is unconsidered in LBP or DLBP. To replenish the missing information in the DLBP features, an additional feature set, features based on the Gabor filter responses are utilized as the supplement to the DLBP features. The Gabor-based features are computed from the normalized average magnitudes of circularly symmetric Gabor filter responses. They are rotation invariant and also less sensitive to histogram equalization. The Gabor-based features are well complemented with the DLBP ones. It is experimentally shown that the fused image features yield significant higher texture classification rates than the mere use of either the DLBP features or the Gabor-based features.

This paper is organized as follows. Section II describes the feature extraction procedure using the dominant local binary patterns (DLBP). Section III then gives the feature extraction procedure using Gabor filters. Section IV presents the experimental results as well as the details of the experiments, including the methods in the comparison study, a brief description of the support vector machines (SVM) classifier, and the experiment setups. Section V gives the conclusions of this paper.

\section{Dominant Local Binary Patterns (DLBP)}

\section{A. An Overview of Local Binary Patterns (LBP)}

The method using local binary patterns (LBPs) is first proposed by Ojala et al. [19] to encode the pixel-wise information in the texture images. At a center pixel $t_{c}$, each neighboring pixel is assigned with a binary label, which can be either "0" or " 1 ," depending on whether the center pixel has higher intensity value than the neighboring pixel (see Fig. 1 for an illustration). The neighboring pixels are the angularly evenly distributed sample points over a circle with radius $R$ centered at the center pixel. The LBP label for that center pixel is given by

$$
L B P_{m, R}=\min _{0 \leq n<m}\left\{\sum_{i=0}^{m-1} u\left(t_{i}-t_{c}\right) 2^{[(i+n) \bmod m]}\right\}
$$

where $t_{c}$ represents the center pixel, $t_{i}$ is the $i$ th neighboring pixel, $i=0, \ldots, m-1, m$ is the total number of neighboring pixels, $R$ is the circle radius which determines how far the neighboring pixels are located away from the center pixel, and $u(x)=$ 1 if $x \geq 0$ else $u(x)=0$. The value of $m$ is assigned according to the value of $R$ as suggested in [19]. In our implementation, $m=8$ when $R=1 ; m=16$ when $R=2$; and $m=24$ when $R=3$. It is noted that computing LBP based on (1) is a rotation invariant operation. Rotating an image causes the circular shifting of the binary labels at locations $t_{0}, t_{1}, \ldots$, and $t_{m-1}$. This shifting effect can be eliminated by finding the minimum value among all possible values of $n$ in (1). This minimum value denotes the rotation invariant LBP at the center pixel $t_{c}$. Furthermore, the absolute pixel intensity information at $t_{c}$ and $t_{i}$

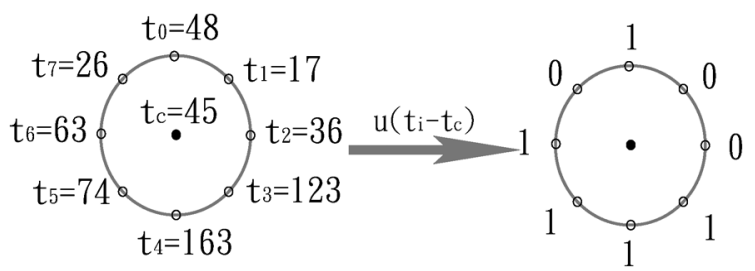

Fig. 1. Example of finding the binary labels of eight circular neighboring pixels at locations $t_{0}, t_{1}, \ldots$, and $t_{7}$, given the center pixel $t_{c} . u\left(t_{i}-t_{c}\right)$ represents a step function, where $u(x)=1$ when $x \geq 0$; else, $u(x)=0$.

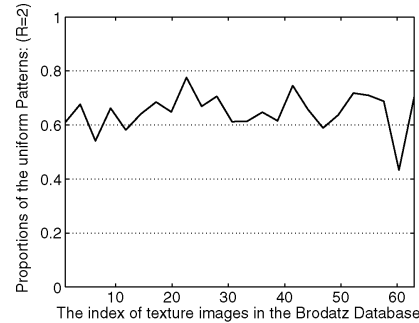

(a)

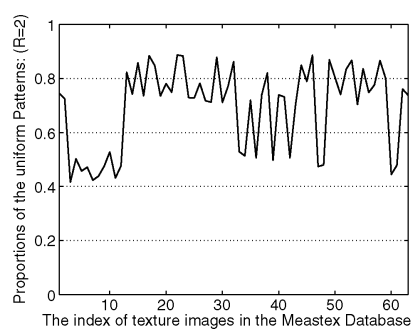

(c)

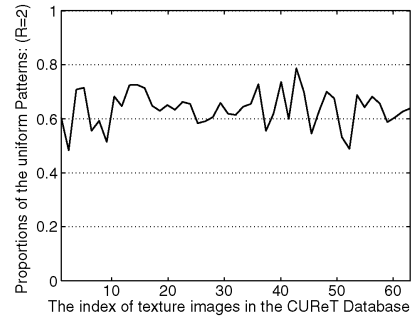

(e)

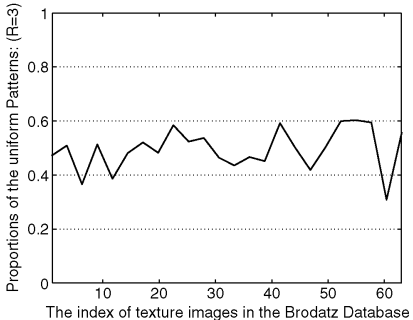

(b)

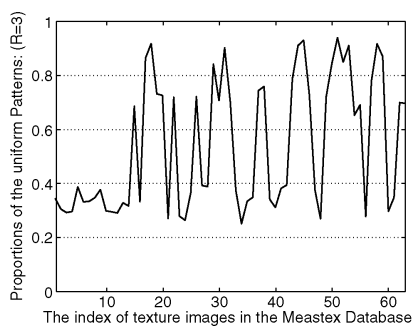

(d)

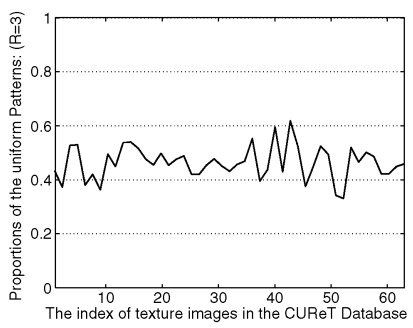

(f)
Fig. 2. Proportions of the uniform LBPs for $R=2$ and $R=3$ in the texture images obtained from the Brodatz database (a), (b), Meastex database (c), (d) and CURet database (e), (f).

is discarded by using the step function $u\left(t_{i}-t_{c}\right)$ in (1) when calculating LBP. Therefore, the LBP operator is not sensitive to histogram equalization.

\section{B. Dominant Local Binary Patterns (DLBP)}

In the conventional LBP method proposed by Ojala et al. [19], only the uniform LBPs are considered. At a pixel, it gives a uniform LBP if the corresponding binary label sequence has no more than two transitions between " 0 " and "1" among all pairs of the adjacent binary labels. For example, the binary label sequences "10001111" and "00011000" are uniform LBPs. But the sequence "01001111" is not a uniform LBP because it has four transitions. In the textures which mostly consist of straight edges or low curvature edges, the uniform LBPs effectively capture the fundamental information of textures. However, in practice, there are some texture images 


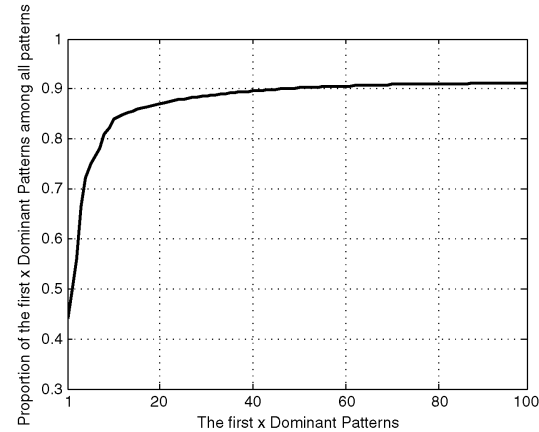

(a)

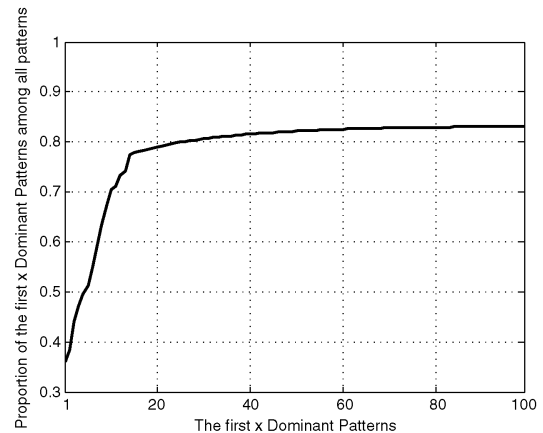

(b)

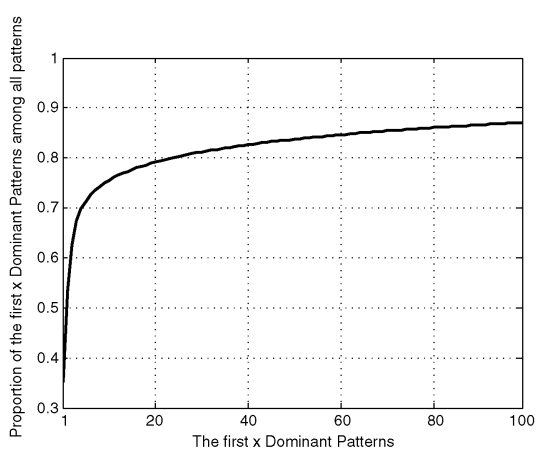

(c)

Fig. 3. Proportions of the first $x$ patterns among all patterns appeared in the texture images of the (a) Brodatz database, (b) Meastex database, and (c) CUReT database.

having more complicated shapes. These shapes can contain high curvature edges, crossing boundaries or corners. Performing texture classification on these textures based on uniform LBPs is possibly problematic. The reason is that the uniform LBPs extracted from such images are not necessary to be the patterns having dominating proportions. For illustration, Fig. 2 shows the proportions of the uniform LBPs extracted from texture images of the Brodatz, Meastex and CURet databases. It is observed that the uniform LBPs are not necessary to occupy the major pattern proportions. Consequently, textural information cannot be effectively represented by solely considering the histogram of the uniform LBPs.

Although utilizing the uniform LBPs is insufficient to capture textural information, we avoid considering all the possible patterns to perform classification. As pointed out by Ojala et al. [19], the occurrence frequencies of different patterns vary greatly and some of the patterns rarely occur in a texture image. The proportions of these patterns are too small and inadequate to provide a reliable estimation of the occurrence possibilities of these patterns.

Therefore, we propose to use dominant local binary patterns (DLBPs) which consider the most frequently occurred patterns in a texture image. It avoids the aforementioned problems encountered by merely using the uniform LBPs or making use of all the possible patterns, as the DLBPs are defined to be the most frequently occurred patterns. In this paper, it will be demonstrated that a minimum set of pattern labels that represents around $80 \%$ of the total pattern occurrences in an image can effectively captures the image textural information for classification tasks. Figs. 3(a)-(c) shows the pattern proportions occupied by different numbers of most frequently occurred patterns in the texture images obtained from the Brodatz, Meastex, and CUReT databases, respectively.

In practice, given a set of training images, the required number of patterns to occupy $80 \%$ pattern occurrences is determined prior to extracting the features of DLBP. This required number of patterns remains the same as the DLBP features are subsequently extracted from the training image set or new testing images. Nonetheless, for two different texture images, the dominant patterns can be of different types. That is, the DLBP approach is not limited to consider only a fixed set of patterns (e.g., uniform patterns). This is distinct to the conventional LBP framework, in which the final feature vector representing an input image is the occurrence histogram of the fixed set of uniform patterns.

To retrieve the DLBP feature vectors from an input image, the pattern histogram which considers all the patterns in the input image is constructed and the histogram bins are sorted in nonincreasing order. Based on the previously computed number of patterns, the occurrence frequencies corresponding to the most frequently occurred patterns in the input image are served as the feature vectors. It is noted that the DLBP feature vectors do not bear information regarding the dominant pattern types, and they only contain the information about the pattern occurrence frequencies. According to the experimental results, omitting the dominant pattern type information in the DLBP feature vectors is not harmful. It is because the $80 \%$ dominant patterns of DLBP is a preponderance of the overall patterns, and the DLBP feature vectors are already very descriptive. It is practically improbable to have two distinct texture types which can resemble dominant pattern proportions of each other.

Without encapsulating the pattern type information, the DLBP features also possess surpassing robustness against image noise, as compared to the conventional LBP features. Under the effect of image noise, the binary label of a neighboring pixel is possible to be flipped by the intensity distortion induced by noise. Flipped binary labels alter the extracted LBPs. As a result, even though some LBPs are computed on the same type of image structures, the extracted LBP type can vary significantly. Thus, the pattern type information is unreliable. In the conventional LBP framework, the pattern types are categorized as uniform patterns or nonuniform patterns. In which, under the effect of image noise, a large amount of useful patterns turns into nonuniform ones that are unconsidered in the conventional LBP method. On the contrary, the DLBP approach processes all $80 \%$ dominant patterns disregarding the pattern types. As reflected by the noisy texture classification experiments in Section IV-D, the number of dominant patterns for classifying noisy textures soars as the noise level increases [see the rows of "Experiment setup \#2 (noisy textures)" in Table I]. The DLBP approach is then capable of capturing more pattern types to deal with the pattern distortion induced by image noise. It in turn retains the high DLBP-based classification accuracies of noise corrupted textures. 
TABLE I

Number of Dominant Patterns of $\mathbf{D L B P}_{R=3}\left(\mathbf{D L B P}_{R=2}\right)$ IN THE EXPERIMENT Setup \#1 AND \#2. In the ROW OF "EXPERIMENT SETUP \#2 (Noisy

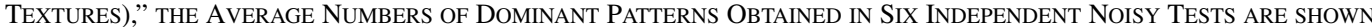

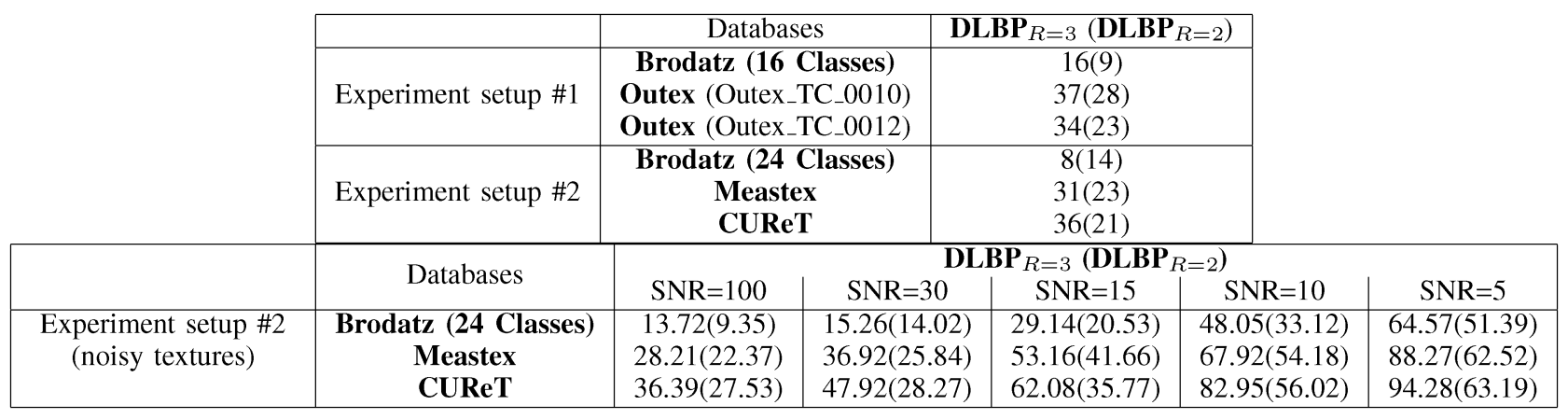

As a summary, the pseudo codes on determining the number of dominant patterns of DLBP and extracting DLBP feature vectors are presented in Algorithm 1 and Algorithm 2, respectively. The computational complexity of DLBP is analyzed as follows. Assuming that $m$ neighboring pixels are considered with respect to each center pixel $t_{c}$ for the evaluation of the (1), and there are $N$ pixels in total for an input image. The time complexity to evaluate (1) is $O(m N)$ because $m$ circular shiftings are required for each pixel, and there are $N$ pixels in total. For the sake of implementation simplicity, an array with $2^{m}$ elements is utilized to represent the pattern histogram (i.e., the length of the occurrence histogram $H$ in Algorithms 1 and 2). Therefore, the sorting of the histogram takes $O\left(2^{m} \log 2^{m}\right)=O\left(m 2^{m}\right)$ time. The time complexity of DLBP is $O(m N)+O\left(m 2^{m}\right)$. Since the value of $m$ is a user specified parameter $(m=16$ or 24 in our experiments), it can be regarded as a constant. Thus, the computation of DLBP is a linear time process.

Algorithm 1 Determining the number of dominant patterns of DLBP

Input: Training image set, and the parameters $m$ and $R$ for DLBP

Output: The required number of patterns $K_{80 \%}$ for $80 \%$ pattern occurrences

1. Initialize $K_{\text {temp }}=0$.

2. FOR each image $I$ in the training image set

3. Initialize the pattern histogram, $H\left[0 \ldots\left(2^{m}-1\right)\right]=0$

4. FOR each center pixel $t_{c} \in I$

5. Compute the pattern label of $t_{c}, L B P_{m, R}(1)$

6. Increase the corresponding bin by $1, H\left[L B P_{m, R}\right]++$

7. END FOR

8. Sort the histogram in descending order

9. Find the number of patterns $k$ for $80 \%$ pattern occurrences in $I$

$$
k=\arg \min _{k}\left(\frac{\sum_{i=0}^{k-1} H[i]}{\sum_{i=0}^{\left(2^{m}-1\right)} H[i]} \geq 80 \%\right) .
$$

10. $K_{\text {temp }}+=k$.

\section{END FOR}

12.

$$
K_{80 \%}=\left\lceil\frac{K_{\text {temp }}}{\text { Number of training images }}\right\rceil
$$

13. Return $K_{80 \%}$

Algorithm 2 Extracting a DLBP feature vector

Input: A training or a testing image $I$, the required number of dominant patterns $K_{80 \%}$, and the parameters $m$ and $R$ for DLBP

Output: The DLBP feature vector corresponding to image $I$

1. Initialize the pattern histogram, $H\left[0 \ldots\left(2^{m}-1\right)\right]=0$

2. FOR each center pixel $t_{c} \in I$

3. Compute the pattern label of $t_{c}, L B P_{m, R}$ (1)

4. Increase the corresponding bin by $1, H\left[L B P_{m, R}\right]++$

\section{END FOR}

6. Sort the histogram in descending order

7. Return $H\left[0 \ldots\left(K_{80 \%}-1\right)\right]$ as the feature vector of DLBP

\section{SUPPLEMENT TO DOMINANT LOCAL BINARY PATTERNS}

DLBP is capable of encoding the pixel-wise information in the texture images. However, it does not take into account the long range pixel interaction that takes place outside the coverage of its circular neighborhood system, which normally has a radius of 2 or 3 pixels. It is noted that capturing such interaction is vital to provide descriptive features for texture classification. In this section, we elaborate the procedure to extract global features from the Gabor filter responses to complement with the DLBP features.

\section{A. Overview of Circularly Symmetric Gabor Filters}

Computing an average response magnitude from a Gabor filtered image is a widely used feature extraction method [2], 
[8], [11], [15], [16], [21], [24], [27]. Since Gabor filters can be viewed as bandpass filters, the average magnitude response for each Gabor filtered image can reflect the image signal power of the corresponding filter passing band. The passing band of a traditional Gabor filter is orientation dependent, which makes it orientation sensitive and rotation variant. To achieve rotation invariance, circularly symmetric Gabor filters are used because each filter has the same passing band in all directions. Therefore, it is capable of extracting information regarding the image signal strength associated with the filter passing band, disregarding the orientation information.

A circularly symmetric Gabor filter is constructed in the Fourier domain and its formulation in the Fourier domain is given by

$$
H(u, v)=\frac{1}{\sqrt{2 \pi} \sigma} \exp \left\{-2\left(\sqrt{u^{2}+v^{2}}-F\right)^{2}(\pi \sigma)^{2}\right\}
$$

where $u$ and $v$ are frequencies in $x$ and $y$ directions, respectively, $\sigma$ controls the passing bandwidth of the filter and $F$ is the center frequency of the filter passing band. With regard to the passing bands of the Gabor filters being utilized, the design strategy of the Gabor filters, presented in [26], is employed. This strategy maximizes the coverage of the frequency domain while minimizing the overlap between filters. In this paper, we use four circularly symmetric Gabor filters, $H_{1}, H_{2}, H_{3}$ and $H_{4}$ with different corresponding center frequencies (measured in cycles/image) $F_{1}=2.0, F_{2}=3.17, F_{3}=5.04$ and $F_{4}=8.0$. The values of $\sigma$ are different for these four filters, $\sigma_{1}=0.4140$, $\sigma_{2}=0.2612, \sigma_{3}=0.1643$, and $\sigma_{4}=0.1035$ so that the upper cut-off frequencies of $H_{1}, H_{2}$ and $H_{3}$ are the same as the lower cut-off frequencies of $\mathrm{H}_{2}, \mathrm{H}_{3}$ and $\mathrm{H}_{4}$ in order to cover sufficient bandwidths in the Fourier domain to perform classification, the design strategy can be referred to [20].

\section{B. Utilizing Gabor Filter Responses}

Conventionally, the averages of the Gabor filter response magnitudes are exploited to represent a Gabor filtered texture image. The average magnitude reflects the signal strength of an image in a particular frequency band. A vital drawback of using the average magnitudes is that it is very sensitive to histogram equalization. This drawback conflicts to the DLBP method, which is invariant to histogram equalization.

To overcome the aforementioned drawback, the Gabor average magnitudes are normalized prior to being utilized as textural features. Suppose $M_{1}, M_{2}, M_{3}$ and $M_{4}$ are the averaged magnitudes of the image filtered by the Gabor filters $H_{1}, H_{2}$, $H_{3}$ and $H_{4}$, respectively, and $M=M_{1}+M_{2}+M_{3}+M_{4}$, the features extracted from the Gabor filter responses are $M_{1} / M$, $M_{2} / M, M_{3} / M$ and $M_{4} / M$.

The above normalized average magnitudes quantify the distribution of the image signal power falling in various passing bands of the corresponding Gabor filters. The image DC signal is out of the passing bands of all the Gabor filters being used. Therefore, the normalized average Gabor filter response magnitudes are invariant to the change of the image intensity average caused by histogram equalization. Meanwhile, the image contrast information is eliminated in the normalized average mag- nitudes which is now invariant to the change of intensity variance [13]. The contrast information is unreliable under the effect of histogram equalization. The normalized average Gabor filter response magnitudes are, therefore, less sensitive to histogram equalization. Thus, it is suitable to provide global information as a supplement to the histogram equalization insensitive DLBP features, which lacks the consideration of distant pixel interactions.

\section{EXPERIMENTS AND RESULTS}

The proposed methods are evaluated against six published approaches under various image conditions. Prior to the discussion of experimental results, we elaborate the implementation details of the experiments. Meanwhile, two sets of experiments have been carried out for testing. The first experiment setup presented in Section IV-C aims at demonstrating that our experimental implementation can replicate the classification results as reported in the state-of-the-art study [19]. This setup utilizes the same texture databases (Brodatz and Outex databases), which were used in the experiments for the evaluation of the LBP approach, see [19] for details of the experiments and databases. The second experiment setup thoroughly and intensively examines the classification performance of various approaches under five different conditions, original textures, randomly rotated textures, histogram-equalized textures, histogram-equalized and randomly rotated textures, and noise corrupted textures.

\section{A. Methods in the Comparison Study}

The classification accuracies of the proposed features have been compared with six well founded feature extraction techniques for texture classification in this section. These six published techniques include.

- Daubechies wavelet transform features (DBWP) [18], [20]: The input texture image is decomposed into three levels. The HH channel of each level is discarded in the experiments because it mainly reflects the noise property in the images. The feature vector has seven dimensions, which consist of the averaged $L_{1}$ norms of the coefficients in the HL and LH channels at all three levels, plus the averaged $L_{1}$ norm of the coefficients in the LL channel.

- Rotation invariant Daubechies wavelet transform features (RDBWP) [20]: This is the rotation invariant version of DBWP described above. The input image is decomposed into three levels. The rotation invariance is achieved by taking an average of the corresponding averaged $L_{1}$ norms of the coefficients in the HL and LH channels at each level. As such, the feature vector has four dimensions.

- Traditional Gabor filters (TGF) [2]: Eight Gabor filters are employed with center frequencies, $F=2.00,3.17,5.04$ and 8.00 and oriented at angles of 0 and 90 degrees to achieve optimal coverage in the Fourier domain. The averaged response magnitude of each filtered image is used as one of the features. The feature vector has eight dimensions.

- Circular Gabor filters (CGF) [8]: Four circularly symmetric Gabor filter with center frequencies $F=2.00$, $3.17,5.04$ and 8.00 are employed. The averaged response 
magnitude of each filtered image is utilized as one of the features. The feature vector has four dimensions.

- Anisotropic circular Gaussian MRFs (ACGMRF) [6]: It is an improved version of the Gaussian Markov random field method [4]. It is rotation invariant and sensitive to directional features. In total, 19 parameters are calculated using the approximated least square estimation based on a third-order neighborhood system with 24 symmetrical orientations.

- Uniform local binary patterns (LBP) [19]: The occurrence histograms of the uniform local binary patterns are computed using $m=8,16,24$ with $R=1,2,3$, respectively. The features are obtained by combining the three sets of features computed over $m=8, R=1 ; m=16, R=2$; and $m=24, R=3$ together. It is claimed to have the best performance of the local binary patterns in the experiments conducted by Ojala et al. [19]. Noted that the local variance measure suggested in [19] is not employed along with the LBP features as this measure is sensitive to the change of illumination conditions of textures [19]. The number of feature dimensions is 54 in LBP: 10 from $R=1$; 18 from $R=2$; and 26 from $R=3$.

- The proposed methods $\left(\mathbf{N G F}, \mathbf{D L B P}_{R=2}, \mathbf{D L B P}_{R=3}\right.$, $\operatorname{DLBP}_{R=2}+\mathbf{N G F}$, and $\left.\operatorname{DLBP}_{R=3}+\mathbf{N G F}\right)$ : The classification performance of the above techniques are studied along with the proposed methods, which embody two components-the DLBP features and the normalized average magnitudes of circularly symmetric Gabor filter responses. For the DLBP approach, we also study the classification rate by using $R=2$ and $R=3$ in (1). With the supplement of Gabor features, there are in total five different types of features based on the proposed methods including normalized Gabor filter response average magnitudes (NGF), the DLBP features with different values of $R\left(\mathbf{D L B P}_{R=2}\right.$ and $\left.\operatorname{DLBP}_{R=3}\right)$ and their fused features $\left(\mathbf{D L B P}_{R=2}+\mathbf{N G F}\right.$ and $\left.\operatorname{DLBP}_{R=3}+\mathbf{N G F}\right)$. It is worth mentioning that the number of image features extracted by the DLBP approach varies as training images change. The lengths of the DLBP feature vectors for different testing cases are listed in Table I.

\section{B. Implementation Details of the Experiments}

Analogous to the works presented in [10] and [14], each feature extraction technique is evaluated and compared with others by passing the features through the same classifier. To appropriately examine the classification performance of various techniques, the support vector machines (SVM) [25], which is well developed to handle classification problems, is employed in this paper as the classifier to perform texture classification based on the extracted features. Based on two-class SVMs, a multiclass classification scheme is constructed by cascading multiple standard two-class SVM classifiers [25] to classify textures with multiple classes.

Given that a texture classification problem involving $N_{T}$ kinds of textures, each two-class SVM classifier is trained by making use of two distinct types of texture images. There are in total $N_{T}\left(N_{T}-1\right) / 2$ two-class SVM classifiers. Each two-class SVM classifier finds the optimal hyperplane to partition the feature space into two halves in order to distinguish the training samples belonging to the two given classes.

Suppose $\vec{x}_{q}$ is the feature vector of the $q$ th training sample, which has a class label $l_{q}$. The values of the class labels are represented as indices $0,1, \ldots, N_{T}-1$. We define a function $y^{i, j}$, which maps each class label $l_{i}$ to a binary value

$$
y^{i, j}(l)= \begin{cases}+1, & \text { if } l=i \\ -1, & \text { if } l=j\end{cases}
$$

and its inverse $Y^{i, j}$, i.e., $Y^{i, j}\left(y^{i, j}(l)\right) \equiv l$. Each SVM classifier predicts whether a new testing sample $\vec{x}_{\text {new }}$ belongs to either class $i$ or class $j$, where $N_{T}>i>j \geq 0$. The class prediction is given as

$$
\begin{aligned}
& \operatorname{Class}^{i, j}\left(\vec{x}_{\text {new }}\right)=Y^{i, j} \\
& \quad \times\left(\operatorname{Sgn}\left(\sum_{\forall q, l_{q} \in\{i, j\}} y\left(l_{q}\right) \alpha_{q}^{i, j} K\left(\vec{x}_{\text {new }}, \vec{x}_{q}\right)+b^{i, j}\right)\right)
\end{aligned}
$$

where $b^{i, j}$ represents the bias parameter of the optimal hyperplane of the SVM dedicated to classify the texture classes $i$ and $j, \alpha_{q}^{i, j}$ are non-negative Lagrangian multipliers for the training sample $q$ used in the SVM associated with the texture classes $i$ and $j$. The values of $b^{i, j}$ and $\alpha_{q}^{i, j}$ are estimated by maximizing the margin of the decision boundary to classify feature samples belonging to class $i$ and samples belonging to class $j$. A predefined constant $C$ is employed to deal with the cases that training samples cannot be well separated by the decision boundary. This parameter is the upper bound of the Lagrangian multipliers, i.e., $0 \leq \alpha_{q}^{i, j} \leq C$. Meanwhile, $K$ is a radial basis kernel function, $K\left(\vec{x}_{i}, \vec{x}_{j}\right)=\exp \left(-\gamma\left\|\vec{x}_{i}-\vec{x}_{j}\right\|^{2}\right)$, where $\|\cdot\|$ is the Euclidean $L_{2}$-Norm and $\gamma$ is a parameter governing the spread of $K$.

Ideally, the SVM classifiers associated with the true class label of a testing sample deliver correct classification results, while the rest of the classifiers randomly report wrong class labels to that sample. As such, the final multiclass classification result can be retrieved by finding the class label, which is mostly reported by all the $N_{T}\left(N_{T}-1\right) / 2$ two-class SVM classifiers.

The experiments are conducted in MatLab version 7.2. A publicly available MatLab SVM Toolbox [17] is employed. The feature vectors are normalized to have zero mean and unit variance along each dimension. The values of the parameters $C$ and $\gamma$ of SVMs are specified using a grid search scheme. In this scheme, the parameters $C$ and $\gamma$ are searched exponentially in the ranges of $\left[2^{-5}, 2^{16}\right]$ and $\left[2^{-15}, 2^{14}\right]$, respectively, with a step size of $2^{1}$ to probe the highest classification rate. The bilinear interpolation method is applied to retrieve the intensity at the positions that are not on the image grid.

\section{Experiment Setup \#1}

In the first experiment setup, we utilized the same set of texture images, which was also used in the performance study reported in [19]. The main reason to carry out this experiment is to demonstrate that our experiment implementation is capable of producing similar testing results as presented in [19].

1) Texture Databases: Brodatz (16 Classes): The sixteen textures in the Brodatz database were downloaded from the 
TABLE II

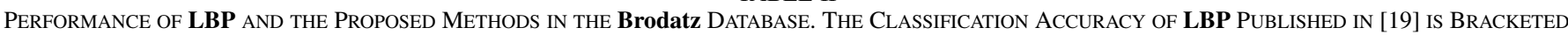
IN THE FIRST COLUMN

\begin{tabular}{|c|c|c|c|c|c|}
\hline \multicolumn{7}{|c|}{ Classification Accuracy \% } \\
\hline LBP & NGF & DLBP $_{R=3}$ & DLBP $_{R=2}$ & $\mathbf{D L B P}_{R=3}+$ NGF & DLBP $_{R=2}+$ NGF \\
\hline $99.7(99.6)$ & 92.7 & 99.4 & 99.8 & 99.3 & 99.8 \\
\hline
\end{tabular}

TABLE III

Performance of LBP and the Proposed Methods in the Outex Database. The Classification Accuracies of LBP Published in [19] are BRACKETED IN THE THIRD COLUMN

\begin{tabular}{|c|c|c|c|c|c|c|c|}
\hline \multirow{2}{*}{ Test suites } & \multirow{2}{*}{$\begin{array}{c}\text { Illumination condition } \\
\text { of testing sets }\end{array}$} & \multicolumn{6}{|c|}{ Classification Accuracy \% } \\
\hline & & LBP & NGF & $\mathbf{D L B P}_{R=3}$ & $\mathbf{D L B P}_{R=2}$ & $\mathbf{D L B P}_{R=3}+\mathbf{N G F}$ & $\mathbf{D L B P}_{R=2}+\mathbf{N G F}$ \\
\hline Outex_TC_00010 & "inca" & $97.6(97.3)$ & 86.2 & 98.1 & 97.7 & 98.2 & 99.1 \\
\hline Outex_TC_00012 & "t184" & $91.3(90.2)$ & 78.3 & 91.6 & 92.1 & 91.6 & 93.2 \\
\hline Outex_TC_00012 & "horizon" & $85.3(84.1)$ & 65.8 & 87.4 & 88.7 & 87.4 & 90.4 \\
\hline
\end{tabular}

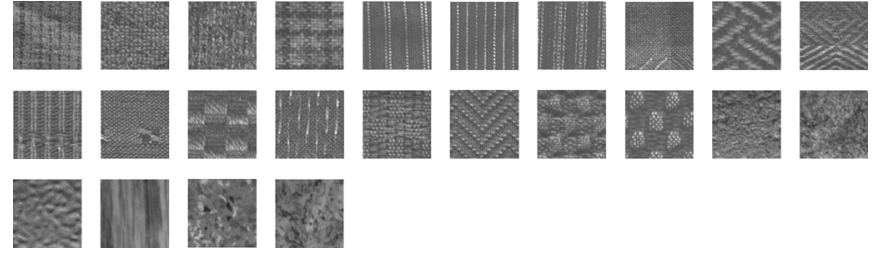

Fig. 4. Texture images with the illumination condition "inca" and zero rotation angle from the 24 classes of textures on Outex database.

website $^{1}$ of the authors of [19]. These sixteen Brodatz textures were previously utilized to conduct the texture classification experiments presented in [19]. There were eight samples for each texture class and the first sample was utilized for training. The rest of the images were served as the testing samples. The training samples were then artificially rotated in four different angles $0^{\circ}, 30^{\circ}, 45^{\circ}$ and $60^{\circ}$, while the testing set consisted of rotation angles $20^{\circ}, 70^{\circ}, 90^{\circ}, 120^{\circ}, 135^{\circ}$, and $150^{\circ}$. As such, there were in total four training images and 42 images presented in the testing set for each texture class. Finally, each image was split into 121 disjoint $16 \times 16$ subimages for both training and testing in the experiments.

Outex: The Outex database contains textural images captured from a wide variety of real materials. The texture images in the Outex database are presented as test suites. The test suites comprise of different textures and are used for evaluating algorithms for various types of texture analysis. In the experiment setup \#1, we made use of the Outex test suites Outex_TC_0010 and Outex_TC_0012, which were created for the classification of rotation invariant textures, and also for the classification of rotation and illumination invariant textures, respectively. In these two test suites, there are 24 classes of texture images captured under various illumination conditions, referred as "inca" (see Fig. 4), "tl84" and "horizon." For each condition, there are nine rotation angles, $0^{\circ}, 5^{\circ}, 10^{\circ}, 15^{\circ}, 30^{\circ}, 45^{\circ}, 60^{\circ}, 75^{\circ}$, and $90^{\circ}$. In a given illumination condition, each rotation angle consists of twenty $128 \times 128$ images for each texture class. The $24 \times 20$ samples with illumination condition "inca" and rotation angle $0^{\circ}$ were adopted as the training data. Three tests using three individual testing sets, which comprised of Outex textures acquired

\footnotetext{
${ }^{1}$ http://www.ee.oulu.fi/mvg/page/image_data.
}

under the illumination conditions "inca," "tl84," and "horizon," were conducted.

2) Classification Accuracy: Tables II (first column) and III (third column) list the classification accuracies of LBP. These accuracies are obtained based on the experiment setup \#1, which follows closely the testing environments presented in [19]. As a comparison, according to [19], the classification accuracies of LBP are listed in the brackets of the same columns. In these tables, by comparing the values of LBP with the corresponding bracketed values, it is observed that our experiment implementation is able to obtain similar testing results of $\mathbf{L B P}$ as compared to those results presented in [19]. The classification accuracies of the proposed methods are also listed in the tables. It is experimentally shown that our methods using both DLBP and Gabor filter responses can achieve high accuracy, as compared with LBP.

\section{Experiment Setup \#2}

In this section, the performance of the proposed features has been evaluated on three databases with large sets of image textures. The three databases are Brodatz album [3], Meastex database [22], and CUReT database [5]. For these databases, classification accuracies have been measured in five different subsets of experiments using original textures (subset \#1), histogram-equalized textures (subset \#2), randomly-rotated textures (subset \#3), histogram-equalized and randomly-rotated textures (subset \#4), and textures corrupted by additive Gaussian noise (subset \#5).

The reasons of performing the above sets of experiments and their details are the followings. First, applying histogram equalization on texture images aims at minimizing the differences in image contrast and illumination condition among different texture samples. However, histogram equalization also alters the image gray level properties of the texture samples, in particular, the mean and standard deviation. As will be shown in the experiment subsets \#2 and \#4, this can affect the texture classification accuracy of the features, which heavily depend on image intensity mean and standard deviation. In the experiment subsets \#2 and \#4, all texture images, including training and testing images, were histogram-equalized by using the same transformation function, $T$, which is given as [7], $s_{k}=T\left(r_{k}\right)=$ 


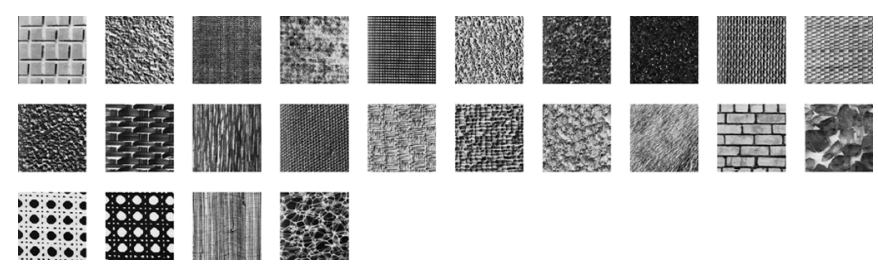

Fig. 5. 24 texture images from the Brodatz album.

$\sum_{j=0}^{k}\left(n_{k} / N\right)$, where $s_{k}$ and $r_{k}$ represent the output and input image intensity levels, $k=0, \ldots, L-1, L$ is the number of intensity levels ( $L=256$ in this paper), $n_{k}$ is the number of pixels having intensity level $r_{k}$, and $N$ denotes the total number of pixels in an image.

Second, randomly rotating the texture samples can help validate the rotation invariance of different features. In the experiment subsets \#3 and \#4, for each database, each texture sample was rotated according to a randomly generated angle. The angle fell uniformly within 0 and 360 degrees. The classification tests for different features were performed on each database and repeated ten times. Then, the mean accuracy and its associated standard deviation were estimated for the experiment subsets \#3 and \#4 using the randomly-rotated textures.

Finally, performing texture classification under a noisy environment can examine the robustness against noise in real world applications of different features. In experiment subset \#5, for each database, each texture sample was corrupted by the zero mean additive Gaussian noise, where its standard deviation was determined according to the corresponding SNR value. The classification tests were conducted on each database and repeated eight times. For each database, the mean and standard deviation of the classification accuracy were obtained for the comparison between different features.

1) Texture Databases: Brodatz (24 Classes): There are twenty four homogeneous texture images selected from the Brodatz album [3] (see Fig. 5). Each texture image has the size of $640 \times 640$ pixels and represents a texture class. In the experiments, each texture image was partitioned into twenty five nonoverlapping sub-images with the size of $128 \times 128$ pixels. For the experiments using the original textures and histogram equalized textures, each $128 \times 128$ sub-image was downsampled to the size of $64 \times 64$ pixels by taking the average between four adjacent pixels. To avoid the boundary problem when the sub-images were rotated, for each $128 \times 128$ sub-image, instead of downsampling, only the center $64 \times 64$ pixels were used in the experiments. Therefore, in all the experiments, twenty five $64 \times 64$ texture samples were generated for each texture class. The SVM classifier was trained by using thirteen samples of each class. The other twelve samples were used for validation.

Meastex: In the Meastex database [22], there are sixty nine texture images selected (see Fig. 6). Each image has the size of $512 \times 512$ pixels. The texture images are categorized into 28 kinds of homogeneous textures. For the experiments using the original textures and histogram equalized textures, each $512 \times$ 512 texture image was partitioned into sixty four nonoverlapping sub-images with the size of $64 \times 64$ pixels. For the experiments using the rotated textures, each texture image was parti-

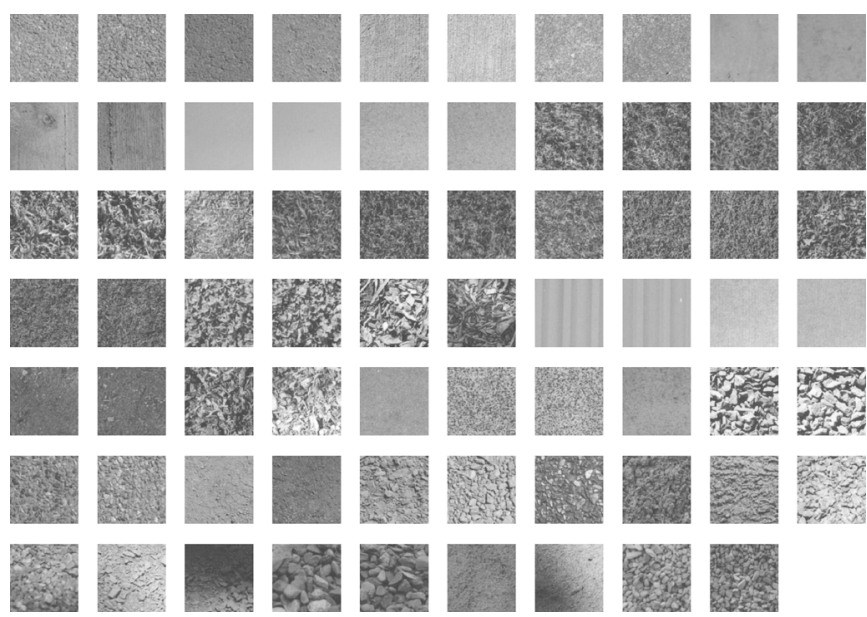

Fig. 6. 69 texture images from the Meastex database.

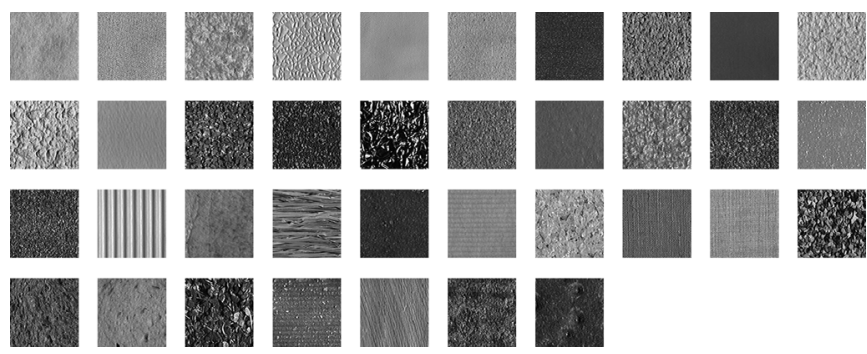

Fig. 7. 47 texture images from the CUReT database.

tioned into sixteen $128 \times 128$ nonoverlapping sub-images. The center $64 \times 64$ pixels were then used. As such, sixteen samples were generated for each texture image for the experiments using rotated textures. In all the experiments, half of the samples were placed in the training set, while the rest of the samples were put in the test-sets.

CUReT: For the CUReT database [5], there are forty seven kinds of homogeneous textures (see Fig. 7). Each kind of textures is represented by one texture image, which has the size of $320 \times 320$ pixels. We partitioned each $320 \times 320$ texture image into nine $106 \times 106$ nonoverlapping sub-images. For the experiments using the original textures and histogram equalized textures, the center $64 \times 64$ pixels were used without the need of interpolation. For the rotated texture-based experiments, the center $64 \times 64$ pixels were used after rotation. In total, nine 64 $\times 64$ samples were generated for each texture image. Five samples were used as the training data, and the other four samples were utilized as the testing data.

\section{E. Classification Accuracy}

The experimental results on classification accuracy are presented in Tables IV-IX. The proposed methods are compared with other six widely used methods, which include DBWP (Row 1), RDBWP (Row 2), TGF (Row 3), CGF (Row 4), ACGMRF (Row 5), and LBP (Row 6).

For the results obtained using NGF alone, see Row 7 in the tables. For the results obtained using DLBP alone with $R=3$ and $R=2$, see $\mathbf{D L B P}_{R=3}$ (Row 8) and $\mathbf{D L B P}_{R=2}$ (Row 9 ), respectively. For the results obtained using both the DLBP 
TABLE IV

Performance of Different Features of $64 \times 64$ Image Resolutions in the Brodatz Database. For Each TeSt (EACH COLumn CoRRESPONDINGLY), the Highest Two MEAn Classification ACCuRACIES ARE Highlighted IN BOLD

\begin{tabular}{|c|c|c|c|c|}
\hline \multirow[b]{2}{*}{ Features } & \multicolumn{4}{|c|}{ Classification Accuracy \% } \\
\hline & $\begin{array}{l}\text { Original } \\
\text { Textures }\end{array}$ & $\begin{array}{l}\text { Histogram-equalized } \\
\text { Textures }\end{array}$ & $\begin{array}{l}\text { Randomly-rotated } \\
\text { Textures }\end{array}$ & $\begin{array}{l}\text { Histogram-equalized \& } \\
\text { Randomly-rotated Textures }\end{array}$ \\
\hline 1. DBWP [18] & 98.15 & 93.52 & $81.13 \pm 5.35$ & $63.56 \pm 5.34$ \\
\hline 2. RDBWP [20] & 90.74 & 79.62 & $90.62 \pm 0.75$ & $77.24 \pm 1.54$ \\
\hline 3. TGF [2] & 98.61 & 91.67 & $78.16 \pm 6.77$ & $67.24 \pm 7.03$ \\
\hline 4. CGF [8] & 85.65 & 67.13 & $83.53 \pm 1.83$ & $57.05 \pm 1.33$ \\
\hline 5. ACGMRF [6] & 95.83 & 93.13 & $93.23 \pm 3.52$ & $85.34 \pm 3.14$ \\
\hline 6. LBP [19] & 97.22 & 96.30 & $92.72 \pm 2.45$ & $91.83 \pm 2.42$ \\
\hline 7. NGF & 81.42 & 78.37 & $80.91 \pm 1.24$ & $74.83 \pm 1.36$ \\
\hline 8. $\operatorname{DLBP}_{R=3}$ & 98.26 & 97.17 & $96.04 \pm 1.14$ & $96.11 \pm 0.38$ \\
\hline 9. $\mathrm{DLBP}_{R=2}$ & 98.49 & 98.49 & $96.18 \pm 1.03$ & $96.25 \pm 1.26$ \\
\hline 10. $\mathrm{DLBP}_{R=3}+\mathrm{NGF}$ & 99.16 & 99.16 & $98.47 \pm 1.17$ & $97.11 \pm 1.23$ \\
\hline 11. $\mathrm{DLBP}_{R=2}+\mathrm{NGF}$ & 99.54 & 99.28 & $99.05 \pm 0.75$ & $98.42 \pm 0.49$ \\
\hline
\end{tabular}

TABLE V

Performance of Different Features of $64 \times 64$ Image Resolution in the Meastex Database. For Each TeSt (EACH Column CoRrespondingly), the HigheSt Two MEAN ClassificATION ACCURACIES ARE Highlighted IN BOLD

\begin{tabular}{|c|c|c|c|c|}
\hline \multirow[b]{2}{*}{ Features } & \multicolumn{4}{|c|}{ Classification Accuracy \% } \\
\hline & $\begin{array}{l}\text { Original } \\
\text { Textures }\end{array}$ & $\begin{array}{c}\text { Histogram-equalized } \\
\text { Textures }\end{array}$ & $\begin{array}{l}\text { Randomly-rotated } \\
\text { Textures }\end{array}$ & $\begin{array}{c}\text { Histogram-equalized \& } \\
\text { Randomly-rotated Textures }\end{array}$ \\
\hline 1. DBWP [18] & 53.80 & 42.58 & $47.15 \pm 6.84$ & $37.28 \pm 6.36$ \\
\hline 2. RDBWP [20] & 44.58 & 32.13 & $44.73 \pm 2.84$ & $34.37 \pm 3.66$ \\
\hline 3. TGF [2] & 57.85 & 47.36 & $46.35 \pm 5.83$ & $44.35 \pm 5.72$ \\
\hline 4. CGF [8] & 51.30 & 42.39 & $53.83 \pm 2.31$ & $40.21 \pm 2.45$ \\
\hline 5. ACGMRF [6] & 64.13 & 64.06 & $60.35 \pm 3.23$ & $50.23 \pm 3.82$ \\
\hline 6. LBP [19] & 54.47 & 52.06 & $57.17 \pm 2.85$ & $54.14 \pm 2.45$ \\
\hline 7. NGF & 46.28 & 43.07 & $45.58 \pm 1.52$ & $41.81 \pm 1.84$ \\
\hline 8. $\operatorname{DLBP}_{R=3}$ & 66.25 & 60.62 & $61.18 \pm 1.08$ & $59.15 \pm 1.18$ \\
\hline 9. $\operatorname{DLBP}_{R=2}$ & 71.36 & 66.82 & $64.07 \pm 0.76$ & $62.83 \pm 1.33$ \\
\hline 10. $\mathrm{DLBP}_{R=3}+\mathrm{NGF}$ & 77.28 & 74.93 & $76.71 \pm 1.79$ & $74.38 \pm 1.13$ \\
\hline 11. $\mathrm{DLBP}_{R=2}+\mathrm{NGF}$ & 81.24 & 79.32 & $81.07 \pm 0.43$ & $80.15 \pm 1.62$ \\
\hline
\end{tabular}

and the Gabor features, see $\mathbf{D L B P} \mathbf{P}_{R=3}+\mathbf{N G F}$ (Row 10) and $\operatorname{DLBP}_{R=2}+$ NGF (Row 11), respectively.

1) Results on the Original Textures: In the second column of Tables IV-VI, the use of dominant patterns instead of uniform patterns is justified by comparing the classification accuracies in the 8th and 9th rows with the sixth row. DLBP methods with $R=2$ and $R=3$ give higher accuracies than the conventional LBP. Furthermore, the improvement of using DLBP instead of conventional LBP for the texture databases Meastex and CUReT (Tables V and VI, respectively) is higher than those for Brodatz (Table IV). It is because the texture images in the Brodatz database contain more obvious edges, and, thus, it has higher proportion of uniform patterns than those in the images of Meastex and CUReT.

2) Rotation Invariance: Experiment subsets \#3 and \#4 have been carried out to study the rotation invariance property of our features and other features. The experimental results are listed in Table IV for the Brodatz album, Table V for the MeasTex database, and Table VI for the CUReT database. In each table, to study the performance regarding the rotation invariance, we can compare the classification accuracies of different features between the "Original Textures" (2nd column) and the "Randomly-rotated Textures" (fourth column). The accuracies can also be compared between the "Histogram-equalized Textures" (third column) and the "Histogram-equalized \& Randomly-rotated Textures" (fifth column). In the tables, the results of other features and our methods are listed in Rows 1-6 and Rows 7-11, respectively.

From the tables, it is observed that our methods (Rows 7-11) do not give significant accuracy drop when image rotation is applied, as compared with other features, e.g., DBWP, TGF and GMRF. In the experiments, the performance of different values of the radius parameter $R$ of DLBP is tested when $R$ is equal to 2 and 3 . The $R=1$ case is not considered in the experiments, as pointed out by [19] that when $R=1$ the neighborhoods are limited to the short distance referring to the center pixel, and, therefore, the pattern's structural property may not be well reflected. Under the same image resolution and image condition, the DLBP features (for both $R=2$ and $R=3$ ) outperform the other six methods in the comparison. As we can see, when $R=2$, fusing the DLBP features with the Gabor-based features yields higher classification accuracy than the case when $R=3$.

3) Less Sensitive to Histogram Equalization: In Table IV, we can compare the accuracies 1) between the "Original Textures" (second column) and "Histogram-equalized Textures" (third column); and 2) between the "Randomly-rotated Textures" (fourth column) and "Histogram-equalized \& Randomly-rotated Textures" (fifth column). It is found that our methods also 
TABLE VI

Performance of Different Features of $64 \times 64$ Image Resolution in the CUReT Database. For Each TeSt (EACh COLumn CoRrespondingly), the Highest Two MEAN ClassifiCATION ACCURACIES ARE HighLIGHTED IN BOLD

\begin{tabular}{|c|c|c|c|c|}
\hline \multirow[b]{2}{*}{ Features } & \multicolumn{4}{|c|}{ Classification Accuracy \% } \\
\hline & $\begin{array}{l}\text { Original } \\
\text { Textures }\end{array}$ & $\begin{array}{c}\text { Histogram-equalized } \\
\text { Textures }\end{array}$ & $\begin{array}{c}\text { Randomly-rotated } \\
\text { Textures }\end{array}$ & $\begin{array}{c}\text { Histogram-equalized \& } \\
\text { Randomly-rotated Textures }\end{array}$ \\
\hline 1. DBWP [18] & 90.43 & 61.17 & $82.43 \pm 6.35$ & $44.74 \pm 6.24$ \\
\hline 2. RDBWP [20] & 85.11 & 39.89 & $82.13 \pm 1.67$ & $42.32 \pm 1.35$ \\
\hline 3. TGF [2] & 76.06 & 44.15 & $65.23 \pm 7.46$ & $28.63 \pm 7.83$ \\
\hline 4. $\mathrm{CGF}[8]$ & 60.64 & 27.66 & $60.35 \pm 1.73$ & $33.45 \pm 1.38$ \\
\hline 5. ACGMRF [6] & 77.13 & 76.85 & $68.33 \pm 7.35$ & $63.45 \pm 7.03$ \\
\hline 6. LBP [19] & 72.87 & 69.15 & $71.87 \pm 2.32$ & $69.47 \pm 1.08$ \\
\hline 7. NGF & 55.42 & 51.85 & $53.97 \pm 2.17$ & $50.23 \pm 1.48$ \\
\hline 8. $\mathrm{DLBP}_{R=3}$ & 83.62 & 78.93 & $83.58 \pm 1.22$ & $76.36 \pm 1.14$ \\
\hline 9. $\operatorname{DLBP}_{R=2}$ & 86.84 & 83.71 & $85.90 \pm 0.82$ & $80.52 \pm 1.03$ \\
\hline 10. $\mathrm{DLBP}_{R=3}+\mathrm{NGF}$ & 90.62 & 86.26 & $88.75 \pm 1.24$ & $85.02 \pm 1.03$ \\
\hline 11. $\operatorname{DLBP}_{R=2}+\mathrm{NGF}$ & 92.77 & 90.83 & $91.62 \pm 1.15$ & $89.26 \pm 0.81$ \\
\hline
\end{tabular}

TABLE VII

Performance of Different Features of $64 \times 64$ Image Resolution in the Brodatz Database in the Additive Gaussian Noise Environment of

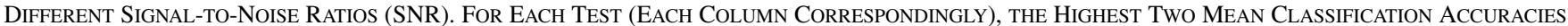
ARE HIGHLIGHTED IN BOLD

\begin{tabular}{|l|ccccc|}
\hline & \multicolumn{5}{|c|}{ Classification Accuracy \% } \\
Features & SNR $=100$ & SNR $=30$ & SNR = 15 & SNR = 10 & SNR = 5 \\
\hline 1. DBWP [18] & $98.61 \pm 0.00$ & $98.61 \pm 0.00$ & $92.39 \pm 1.91$ & $84.25 \pm 4.33$ & $63.32 \pm 7.04$ \\
2. RDBWP [20] & $92.05 \pm 0.45$ & $91.35 \pm 0.35$ & $85.18 \pm 0.64$ & $81.15 \pm 5.35$ & $64.39 \pm 5.27$ \\
3. TGF [2] & $96.15 \pm 1.05$ & $96.05 \pm 1.16$ & $86.04 \pm 3.58$ & $78.06 \pm 7.59$ & $52.05 \pm 9.24$ \\
4. CGF [8] & $90.44 \pm 1.24$ & $91.35 \pm 0.79$ & $84.93 \pm 4.59$ & $73.29 \pm 8.41$ & $42.49 \pm 10.35$ \\
5. ACGMRF [6] & $94.25 \pm 1.26$ & $94.38 \pm 1.05$ & $82.50 \pm 4.53$ & $65.41 \pm 7.37$ & $45.38 \pm 8.36$ \\
6. LBP [19] & $98.11 \pm 0.25$ & $98.04 \pm 0.21$ & $91.35 \pm 2.49$ & $74.85 \pm 5.43$ & $52.45 \pm 5.38$ \\
\hline 7. NGF & $87.62 \pm 0.68$ & $84.94 \pm 0.83$ & $79.62 \pm 1.49$ & $69.65 \pm 4.15$ & $45.92 \pm 5.63$ \\
8. DLBP $R=3$ & $97.38 \pm 0.41$ & $98.04 \pm 1.36$ & $94.02 \pm 1.88$ & $85.06 \pm 6.02$ & $63.84 \pm 6.70$ \\
9. DLBP $R=2$ & $98.34 \pm 0.16$ & $97.92 \pm 0.64$ & $95.72 \pm 1.37$ & $87.72 \pm 3.84$ & $70.03 \pm 5.91$ \\
10. DLBP $R=3+$ NGF & $\mathbf{9 9 . 2 8} \pm \mathbf{0 . 0 0}$ & $\mathbf{9 8 . 6 1} \pm \mathbf{0 . 2 9}$ & $\mathbf{9 3 . 6 2} \pm \mathbf{0 . 7 8}$ & $\mathbf{9 1 . 4 7} \pm \mathbf{5 . 0 2}$ & $\mathbf{7 8 . 2 8} \pm \mathbf{6 . 3 1}$ \\
11. DLBP $R=2+$ NGF & $\mathbf{9 9 . 3 5} \pm \mathbf{0 . 0 0}$ & $\mathbf{9 9 . 3 1} \pm \mathbf{0 . 0 0}$ & $\mathbf{9 5 . 7 7} \pm \mathbf{0 . 9 7}$ & $\mathbf{9 2 . 3 3} \pm \mathbf{4 . 6 5}$ & $\mathbf{8 3 . 8 4} \pm \mathbf{4 . 4 8}$ \\
\hline
\end{tabular}

do not have significant accuracy drop. LBP, DLBP and NGF are robust to histogram equalization. Obviously, the uniform binary patterns and nonuniform binary patterns of $\mathbf{L B P}$ and DLBP are not affected by histogram equalization, which is an intensity monotonic increasing transform. The normalized average Gabor filter responses magnitudes are more robust than that of plain Gabor average response-based features, i.e., TGF and CGF.

For more challenging databases such as Meastex and CUReT, it is observed that the proposed method is less sensitive to histogram equalization. As shown in Tables V and VI, the classification rates of the combined features drop less than $4 \%$ after performing histogram equalization on the textures. It is also observed that LBP and ACGMRF are robust to histogram equalization, as compared with their performance under the original texture environment. However, we should bear in mind that the classification accuracy of the proposed method is much higher than those of both LBP and ACGMRF.

4) Robustness to Noise: In this section, we evaluate the performance of various methods under the noisy environment. In the experiment subset \#5, the original texture images are added with additive Gaussian noise with different signal-to-noise ratios (SNR). The classification process is performed 6 times independently in the additive Gaussian noise environment. Then the average classification accuracies and the standard deviations are calculated for each database.

As we can see, all approaches maintain their performances in all databases when SNR $>=30$. It is reflected by the high average classification accuracies and small standard deviation. The reason is that in such cases, the noise power is negligible compared to the texture image signal power. The textures are not significantly distorted. However, when the SNR value drops below 30, the noise power rises so that different methods have considerable declines of accuracies.

In the Brodatz database, by combining the DLBP and Gabor features, it is more robust against noise as they capture both the local and global texture information. Even though in the toughest situation ( $\mathrm{SNR}=5$ ), the proposed method effectively extract the existing texture information to deliver promising classification rates. The proposed method achieves moderate classification accuracy $(83.84 \%)$ in the toughest case as shown in Table VII. In the Meastex database, as a challenging database, the classification abilities of various methods reduce significantly when SNR falls below 15 (Table VIII). In contrast, the proposed method outperforms other approaches with a barely moderate accuracy. On the other hand, for the CUReT database, it contains the largest number of classes which causes higher risk of mis-classification than other databases mentioned 
TABLE VIII

Performance of Different Features of $64 \times 64$ Image Resolution in the Meastex Database IN THE AdDitive GaUSSiAn Noise EnVIRONMENT OF

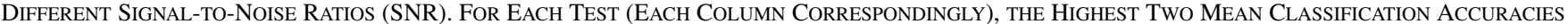
ARE HIGHLIGHTED IN BOLD

\begin{tabular}{|c|c|c|c|c|c|}
\hline \multirow[b]{2}{*}{ Features } & \multicolumn{5}{|c|}{ Classification Accuracy \% } \\
\hline & $\mathrm{SNR}=100$ & $\mathrm{SNR}=30$ & $\mathrm{SNR}=15$ & $\mathrm{SNR}=10$ & $\mathrm{SNR}=5$ \\
\hline 1. DBWP [18] & $50.74 \pm 0.00$ & $50.74 \pm 0.00$ & $41.15 \pm 5.32$ & $35.39 \pm 5.83$ & $23.58 \pm 6.82$ \\
\hline 2. RDBWP [20] & $42.54 \pm 0.00$ & $42.54 \pm 0.00$ & $35.39 \pm 4.33$ & $27.49 \pm 5.18$ & $17.06 \pm 5.21$ \\
\hline 3. TGF [2] & $55.37 \pm 1.03$ & $55.49 \pm 1.24$ & $26.05 \pm 6.39$ & $14.54 \pm 7.32$ & $16.28 \pm 7.28$ \\
\hline 4. CGF [8] & $51.30 \pm 0.00$ & $51.30 \pm 0.00$ & $21.48 \pm 9.06$ & $11.49 \pm 10.28$ & $13.58 \pm 11.48$ \\
\hline 5. ACGMRF [6] & $59.35 \pm 0.49$ & $58.48 \pm 0.74$ & $27.66 \pm 8.19$ & $9.05 \pm 10.05$ & $12.64 \pm 8.50$ \\
\hline 6. LBP [19] & $58.31 \pm 0.00$ & $58.26 \pm 0.24$ & $23.48 \pm 4.78$ & $15.28 \pm 4.25$ & $14.30 \pm 5.28$ \\
\hline 7. NGF & $46.83 \pm 0.00$ & $43.98 \pm 0.07$ & $23.78 \pm 4.83$ & $15.26 \pm 6.15$ & $12.84 \pm 7.23$ \\
\hline 8. $\mathrm{DLBP}_{R=3}$ & $67.92 \pm 0.00$ & $64.46 \pm 0.48$ & $40.72 \pm 6.84$ & $33.37 \pm 6.05$ & $21.40 \pm 5.93$ \\
\hline 9. $\operatorname{DLBP}_{R=2}$ & $71.57 \pm 0.02$ & $70.15 \pm 0.25$ & $45.72 \pm 5.29$ & $38.34 \pm 4.48$ & $25.09 \pm 6.11$ \\
\hline 10. $\operatorname{DLBP}_{R=3}+\mathrm{NGF}$ & $83.18 \pm 0.02$ & $83.28 \pm 0.02$ & $51.64 \pm 4.37$ & 41.67士5.58 & $27.38 \pm 6.82$ \\
\hline 11. $\mathrm{DLBP}_{R=2}+\mathrm{NGF}$ & $85.82 \pm 0.00$ & $\mathbf{8 5 . 8 2} \pm \mathbf{0 . 0 0}$ & $57.83 \pm 3.92$ & $44.29 \pm 6.27$ & $31.85 \pm 7.03$ \\
\hline
\end{tabular}

TABLE IX

Performance of Different Features of $64 \times 64$ ImAge Resolution in the CUReT Database in the AdDitive Gaussian Noise EnVIRONMENT OF

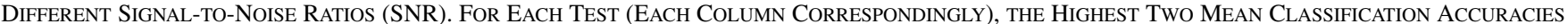
ARE HIGHLIGHTED IN BOLD

\begin{tabular}{|l|ccccc|}
\hline & \multicolumn{5}{|c|}{ Classification Accuracy \% } \\
Features & SNR $=100$ & SNR $=30$ & SNR $=15$ & SNR $=10$ & SNR $=5$ \\
\hline 1. DBWP [18] & $88.37 \pm 1.23$ & $85.33 \pm 1.49$ & $80.38 \pm 6.21$ & $71.40 \pm 8.44$ & $63.40 \pm 7.49$ \\
2. RDBWP [20] & $83.46 \pm 1.04$ & $82.26 \pm 1.59$ & $74.87 \pm 6.36$ & $62.84 \pm 7.31$ & $51.19 \pm 8.39$ \\
3. TGF [2] & $60.79 \pm 1.25$ & $62.69 \pm 1.40$ & $51.80 \pm 8.05$ & $43.67 \pm 11.36$ & $46.41 \pm 9.59$ \\
4. CGF [8] & $52.94 \pm 1.68$ & $53.39 \pm 1.70$ & $52.36 \pm 10.35$ & $46.39 \pm 13.48$ & $46.74 \pm 11.49$ \\
5. ACGMRF [6] & $60.90 \pm 1.50$ & $57.54 \pm 1.69$ & $51.36 \pm 9.95$ & $47.05 \pm 8.41$ & $47.08 \pm 10.36$ \\
6. LBP [19] & $72.21 \pm 1.82$ & $71.24 \pm 1.34$ & $50.33 \pm 7.46$ & $36.40 \pm 6.39$ & $28.48 \pm 6.43$ \\
\hline 7. NGF & $47.86 \pm 1.24$ & $45.49 \pm 1.75$ & $43.14 \pm 3.47$ & $41.63 \pm 4.82$ & $40.06 \pm 5.74$ \\
8. DLBP $R=3$ & $84.52 \pm 0.84$ & $83.36 \pm 1.38$ & $74.14 \pm 4.68$ & $65.81 \pm 6.94$ & $47.46 \pm 7.19$ \\
9. DLBP $R=2$ & $87.82 \pm 0.03$ & $85.28 \pm 0.21$ & $79.44 \pm 4.08$ & $68.73 \pm 6.31$ & $47.72 \pm 4.21$ \\
10. DLBP $R=3$ + NGF & $\mathbf{9 2 . 9 5} \pm \mathbf{0 . 0 2}$ & $\mathbf{9 2 . 0 7} \pm \mathbf{0 . 0 1}$ & $\mathbf{8 8 . 9 4} \pm \mathbf{2 . 2 8}$ & $\mathbf{8 1 . 0 7} \pm \mathbf{5 . 2 8}$ & $\mathbf{6 6 . 8 4} \pm \mathbf{6 . 0 2}$ \\
11. DLBP $R=2+$ NGF & $\mathbf{9 6 . 1 7} \pm \mathbf{0 . 0 0}$ & $\mathbf{9 5 . 7 8} \pm \mathbf{0 . 0 2}$ & $\mathbf{9 2 . 8 1} \pm \mathbf{4 . 5 7}$ & $\mathbf{8 6 . 0 6} \pm \mathbf{4 . 2 3}$ & $\mathbf{7 1 . 2 8} \pm \mathbf{4 . 1 9}$ \\
\hline
\end{tabular}

above. High mis-classification risk limits the classification performances of different techniques. The proposed method outperforms other methods in the noisy cases (Table IX). Meanwhile, it is able to achieve mediocre classification accuracies (66.84\% and $71.28 \%$ for $R=3$ and $R=2$, respectively, see Table IX) when the SNR is 5.

\section{DisCUSSIONS AND CONCLUSIONS}

This paper proposes the dominant local binary patterns (DLBP) as a texture classification approach. The DLBP approach on one side guarantees to be able to represent the dominant patterns in the texture images. On the other side, it retains the rotation invariant and histogram equalization invariant properties of the conventional LBP approach. It is simple and computationally efficient. Regarding the selection of the first $80 \%$ most frequently appeared patterns as features after the pattern frequency histogram is constructed and sorted, as demonstrated in Section II. It is experimentally shown that it gives good texture classification results.

The global features extracted by using the circularly symmetric Gabor filter responses encapsulate the spatial relationships between distant pixels. The global features extracted from Gabor filter responses are rotation invariant and less sensitive to histogram equalization. They, therefore, complement with the DLBP local features as mirrored by the experimental results.

As demonstrated by the experimental results, it is found that when $R=2$, the DLBP and the Gabor-based features achieve the best classification results. The robustness of the proposed approach to image histogram equalization, random rotation and noise is validated by the experiments carried out under five different image conditions: original textures; histogram equalized textures; randomly rotated textures and; histogram equalized and randomly rotated textures; textures with additive Gaussian noise.

Moreover, its excellent classification performance is demonstrated in three databases, which contain low image resolution textures (Brodatz), similar appearance textures (Meastex), texture set with large number of classes (CUReT). Besides, the performance of the proposed method is compared with six widely used image features. The experimental results show that the proposed method outperforms the other image features in terms of the classification rates in various image conditions.

\section{REFERENCES}

[1] H. Arof and F. Deravi, "Circular neighborhood and 1-D DFT features for texture classification and segmentation," Proc. IEE Vision, Image and Signal Process., vol. 145, no. 3, pp. 167-172, Jun. 1998. 
[2] A. C. Bovik, M. Clark, and W. S. Geisler, "Multichannel texture analysis using localized spatial filters," IEEE Trans. Pattern Anal. Mach. Intell., vol. 12, no. 1, pp. 55-73, Jan. 1990.

[3] P. Brodatz, Textures: A Photographic Album for Artists and Designers. New York: Dover, Jun. 1966.

[4] R. Chellappa and S. Chatterjee, "Classification of textures using Gaussian Markov random fields," IEEE Trans. Acoust., Speech, Signal Process., vol. ASSP-33, no. 4, pp. 959-963, Apr. 1985.

[5] K. Dana, B. Ginneken, S. Nayar, and J. Koenderink, "Reflectance and texture of real-world surfaces," ACM Trans. Graph., vol. 18, no. 1, pp. 1-34, Jan. 1999.

[6] H. Deng and D. A. Clausi, "Gaussian MRF rotation-invariant features for image classification," IEEE Trans. Pattern Anal. Mach. Intell., vol. 26, no. 7, pp. 951-955, Jul. 2004.

[7] R. C. Gonzalez and R. E. Woods, Digital Image Processing. Englewood Cliffs, NJ: Prentice-Hall, 2002.

[8] G. M. Haley and B. S. Manjunath, "Rotation-invariant texture classification using a complete space-frequency model," IEEE Trans. Image Process., vol. 8, no. 2, pp. 255-269, Apr. 1999.

[9] X. Huang, S. Z. Li, and Y. Wang, "Shape localization based on statistical method using extended local binary pattern," in Proc. 3rd Int. Conf. Image and Graphics, 2004, pp. 184-187.

[10] K. Jafari-Khouzani and H. Soltanian-Zadeth, "Radon transform orientation estimation for rotation invariant texture analysis," IEEE Trans. Pattern Anal. Mach. Intell., vol. 27, pp. 1004-1008, 2005.

[11] L. M. Kaplan, "Extended fractal analysis for texture classification and segmentation," IEEE Trans. Image Process., vol. 8, pp. 1572-1585, 1999.

[12] R. L. Kashyap and A. Khotanzad, "A model-based method for rotation invariant texture classification," IEEE Trans. Pattern Anal. Mach. Intell., vol. 8, no. 7, pp. 472-481, Jul. 1986.

[13] M. W. K. Law and A. C. S. Chung, "Weighted local variance based edge detection and its application to vascular segmentation in magnetic resonance angiography," IEEE Trans. Med. Imag., vol. 26, no. 9, pp. 1224-1241, Sep. 2007.

[14] S. Li and J. Shawe-Taylor, "Comparison and fusion of multiresolution features for texture classification," Pattern Recognit. Lett., vol. 26, pp. 633-638, 2005.

[15] C. Liu and H. Wechsler, "Gabor feature based classification using the enhanced fisher linear discriminant model for face recognition," IEEE Trans. Image Process., vol. 11, pp. 467-476, 2002.

[16] C. Liu and H. Wechsler, "Independent component analysis of Gabor features for face recognition," IEEE Trans. Neural Netw., vol. 14, pp. 919-928, 2003

[17] J. Ma, Y. Zhao, and S. Ahalt, OSU SVM Classifier Matlab Toolbox, ver. 3.00 [Online]. Available: http://www.ece.osu.edu/maj/osu svm/osu svm3.00.zip

[18] S. G. Mallat, "A theory for multiresolution signal decomposition: The wavelet representation," IEEE Trans. Pattern Anal. Mach. Intell., vol. 11, no. 7, pp. 674-693, Jul. 1989.

[19] T. Ojala, M. Pietikainen, and T. Maenpaa, "Multiresolution gray-scale and rotation invariant texture classification with local binary patterns," IEEE Trans. Pattern Anal. Mach. Intell., vol. 24, no. 7, pp. 971-987, Jul. 2002.

[20] R. Porter and N. Canagarajah, "Robust rotation-invariant texture classification: Wavelet, Gabor filter and GMRF based schemes," IEE Proc. Vis., Image, Signal Process., vol. 144, no. 3, pp. 180-188, 1997.

[21] N. Qaiser, M. Hussain, A. Hanif, S. M. J. Rizvi, and A. Jalil, "Fusion of optimized moment based and Gabor texture features for better texture classification," in Proc. 8th Int. Multitopic Conf., 2004, pp. 41-48.

[22] G. Smith, MeasTex Image Texture Database and Test Suite Centre for Sensor Signal and Information Processing., Univ. Queensland, Jan. 1998.
[23] J. Weszka, C. R. Dyer, and A. Rosenfeld, "A comparative study of textures measures for terrain classification," IEEE Trans. Syst., Man, Cybern., vol. SMC-6, pp. 269-285, 1976.

[24] H. Yao, B. Li, and W. Cao, "Remote sensing imagery retrieval based-on Gabor texture feature classification," in Proc. 7th Int. Conf. Signal Processing, 2004, vol. 1, pp. 733-736.

[25] J. Zhang, T. N. Tan, and L. Ma, "Support-vector network," Mach. Learn., pp. 901-904, 1995.

[26] J. Zhang, T. N. Tan, and L. Ma, "Invariant texture segmentation via circular Gabor filters," in Proc. 16th IEEE Int. Conf. Pattern Recognition, 2002, pp. 901-904.

[27] C. G. Zhao, H. Y. Cheng, Y. L. Huo, and T. G. Zhuang, "Liver CT-image retrieval based on Gabor texture," in Proc. 26th Annu. Int. Conf. Engineering in Medicine and Biology Society, 2004, vol. 1, pp. 1491-1494.

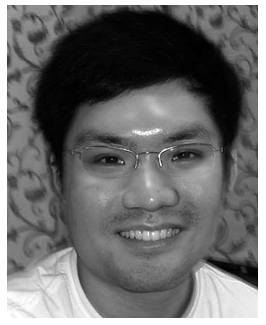

S. Liao received the B.Eng. degree (first class honor and academic award medal) in computer engineering and the M.Phil. degree in computer science from The Hong Kong University of Science and Technology in 2005 and 2007, respectively, where he is currently pursuing the Ph.D. degree in computer science and engineering.

His research interests include medical image registration, texture analysis, and face recognition. In particular, he has worked on new feature extraction and image representation methods.

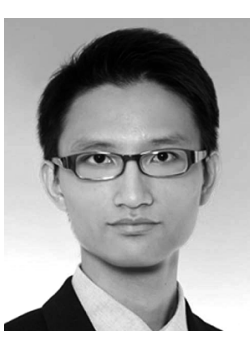

Max W. K. Law received the B.Eng. degree in computer engineering and the M.Phil. degree in computer science in 2004 and 2006, respectively, from The Hong Kong University of Science and Technology. He is currently pursuing the Ph.D. degree in computer science and engineering at The Hong Kong University of Science and Technology.

His research interests include medical image processing and analysis, vascular segmentation, medical image registration, edge detection, and texture recognition.

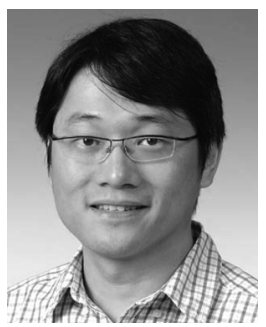

Albert C. S. Chung received the B.Eng. degree (first class Honors) in computer engineering from The University of Hong Kong in 1995 and the M.Phil. degree in computer science from The Hong Kong University of Science and Technology in 1998.

He joined the Medical Vision Laboratory, University of Oxford, Oxford, U.K., as a doctoral research student with a Croucher Foundation scholarship and graduated in 2001. He was a Visiting Scientist at the Artificial Intelligence Laboratory, Massachusetts Institute of Technology, Cambridge, in 2001. He is currently an Associate Professor with the Department of Computer Science and Engineering, The Hong Kong University of Science and Technology. His research interests include medical image analysis, image processing, and computer vision.

Dr. Chung won the 2002 British Machine Vision Association Sullivan Thesis Award for the best doctoral thesis submitted to a U.K. university in the field of computer or natural vision. 